\title{
Isomerization of urocanic acid by ultraviolet radiation and its role in modulation of
}

skin microbiome, antimicrobial peptides, and immune function.

VijayKumar Patra $^{1}$, Mina Bashir ${ }^{3}$, Meghana Somlapura ${ }^{2}$, Harald C. Köfeler ${ }^{4}$, Thomas Pieber ${ }^{3}$, Peter Wolf ${ }^{1}$

\section{Introduction:}

Urocanic acid (UCA) is present locally in the stratum corneum of the skin in its trans isoform (transUCA)

Upon ultraviolet-radiation (UV-R) trans-UCA is isomerized to cis-UCA. ${ }^{1}$

cis-UCA is known to induce immune suppression via $5-\mathrm{HT}_{2} \mathrm{~A}$ receptor, serotonin signaling and various other pathways. ${ }^{2}$

Since skin microbiome is established all over the surface of the skin, we investigated the effects of cis-UCA on the skin microbiome and antimicrobial peptides (AMPs) expression and further looked into the immune modulation using mouse models.

\section{Results:}

\section{PUVA and UV-B isomerizes trans-UCA to cis-UCA.}

UV-B

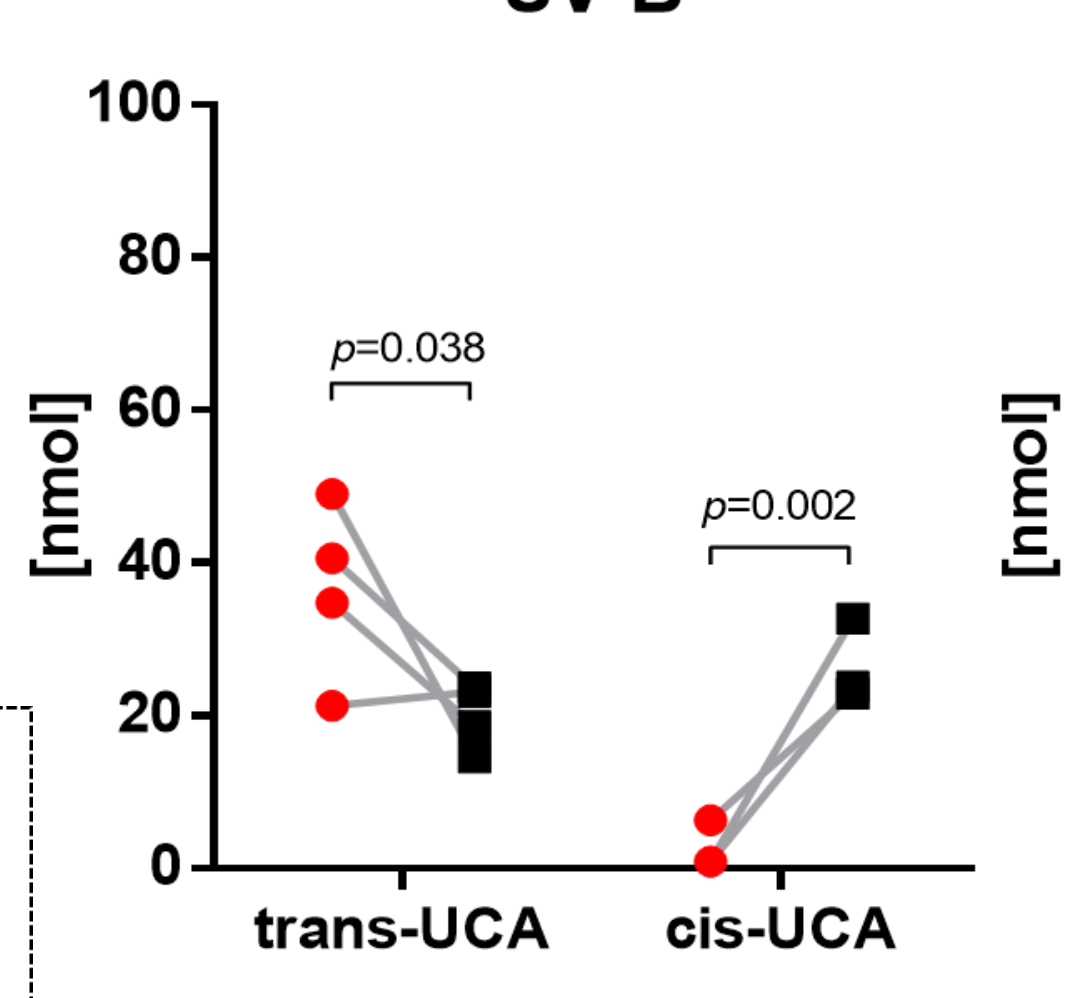

8-MOP

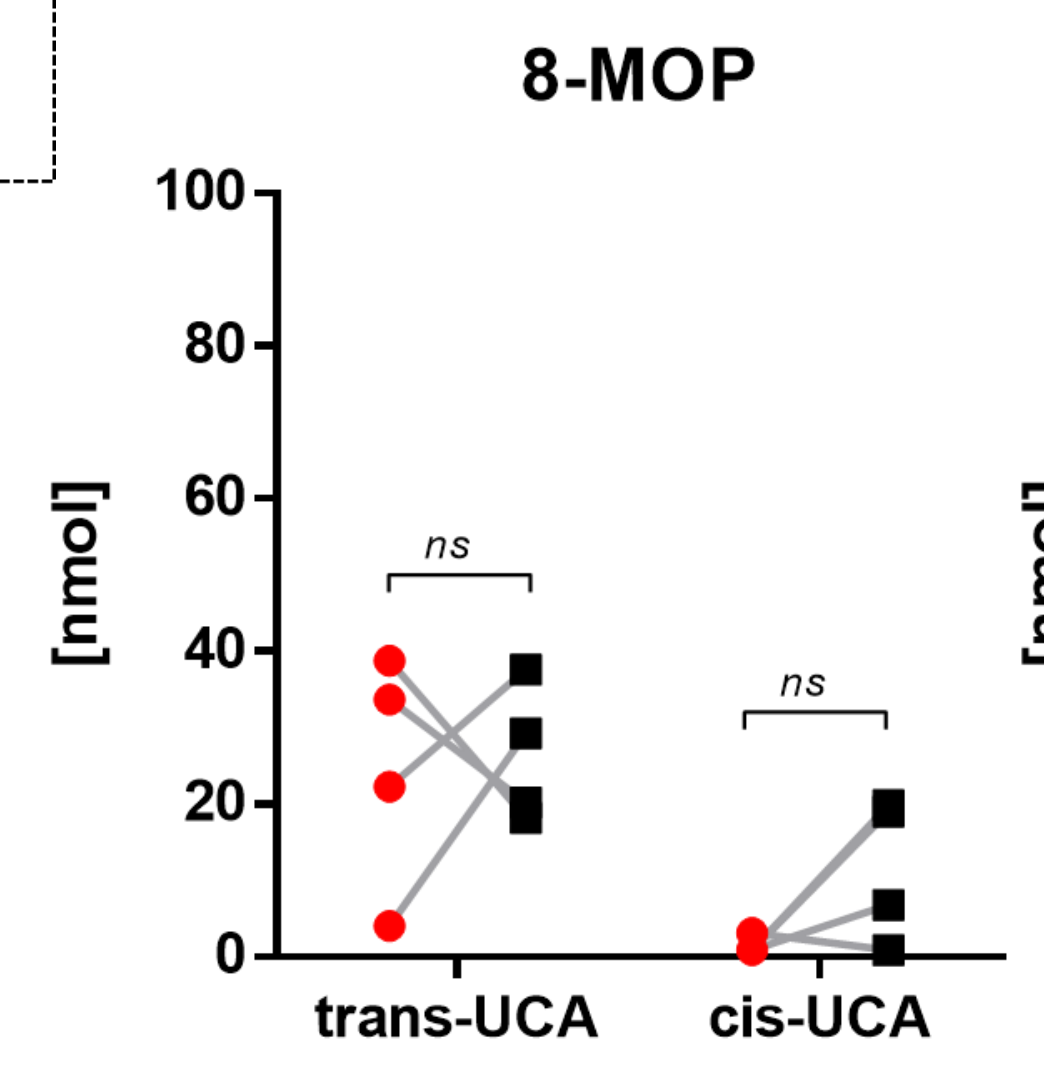

PUVA
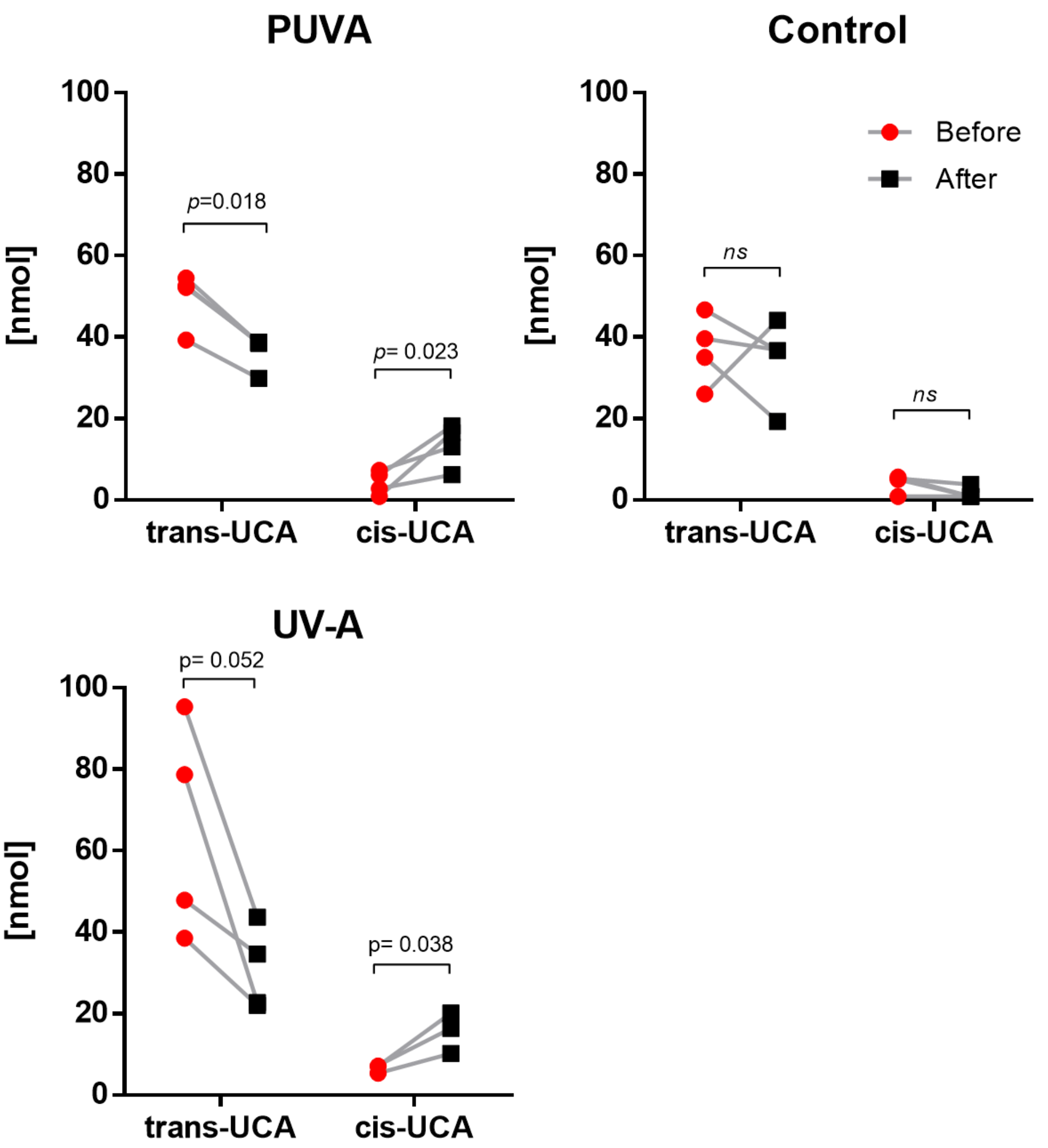

Fig 1: PUVA $\left(1125 \mathrm{~mJ} / \mathrm{cm}^{2}\right)$, UV-A $\left(1125 \mathrm{~mJ} / \mathrm{cm}^{2}\right)$ and UV-B $\left(618 \mathrm{~mJ} / \mathrm{cm}^{2}\right)$ significantly isomerizes trans to cis-UCA, but not or psoralen (8-MOP)

T-test (Holm-Sidak method, with alpha $=0.5000 \%$ ).

\section{2. cis-UCA modulates microbes on the skin and AMP gene expression at $8 \mathrm{~h}$.}
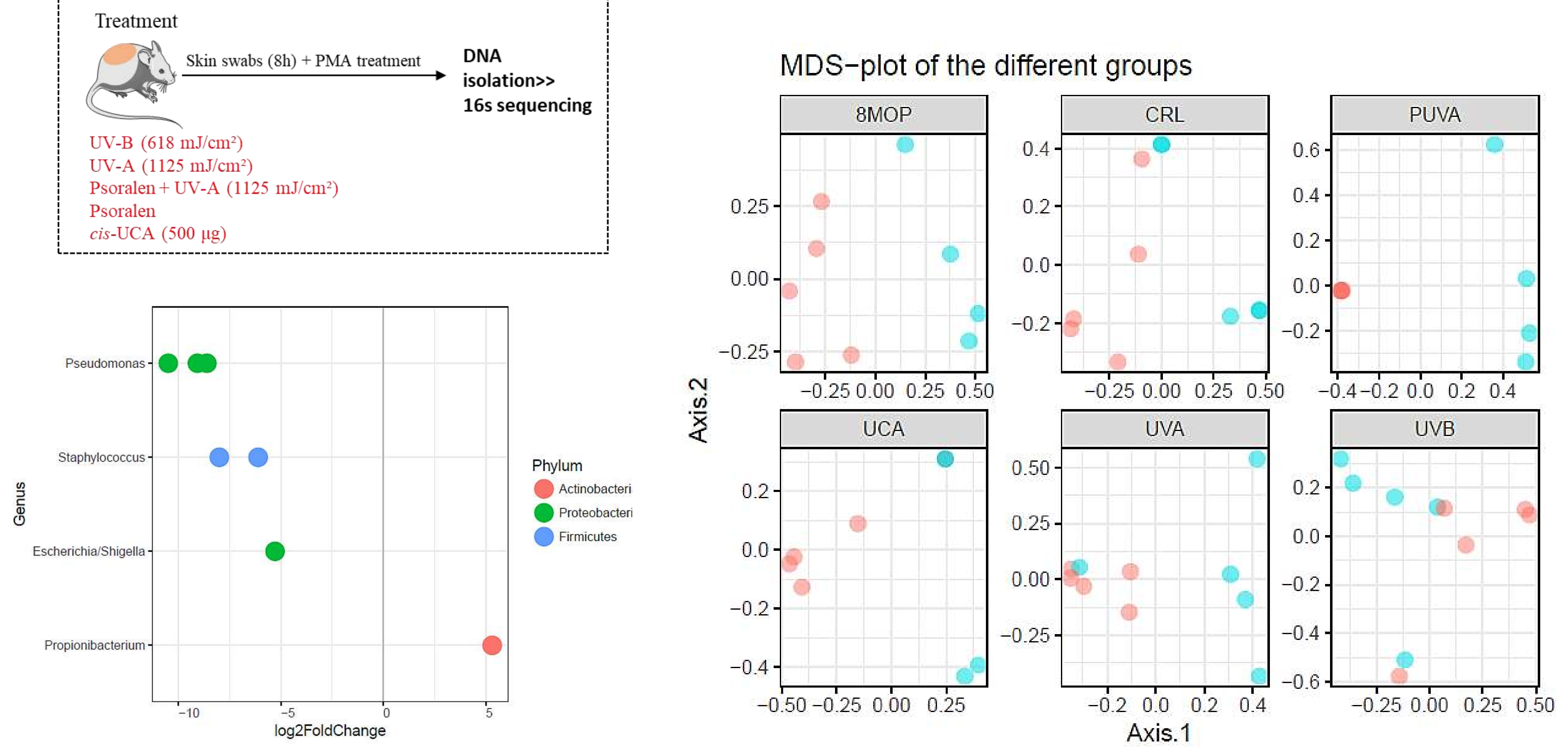

2 (a): Modulation of skin microbiome by cis-UCA (500 $\mu \mathrm{g}$ ) and UV-R at $8 \mathrm{~h}$. MDS plots show a distinct separation between skin microbiome before and after treatment, notably for cis-UCA. Furthermore cis-UCA significantly increase abundance of Propionibacterium, and reduces Pseudomonas, Staphylococcus and Escherichia/Shigella species.

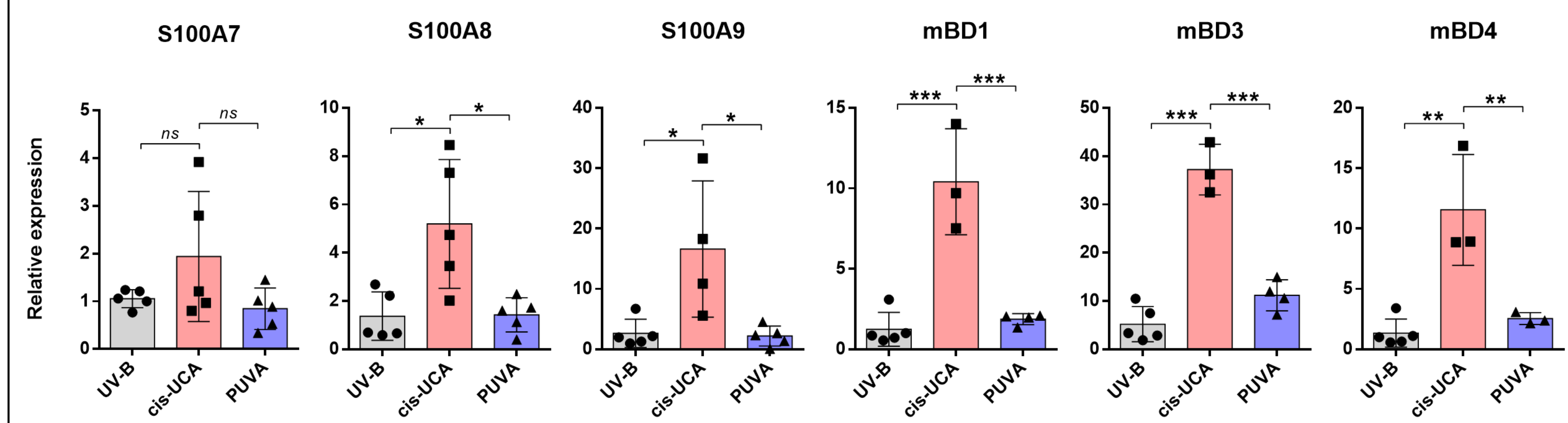

Fig 2 (b): cis-UCA $(500 \mu \mathrm{g})$ increases gene expression of various AMPs at 8h. cis-UCA significantly increased gene expression of calcium binding proteins (S100A8 and S100A9), along with beta-defensins $(m B D-1,-2 \&-3)$

\section{Results:}

3. cis-UCA modulates microbes and AMPs on the skin at $24 \mathrm{~h}$.
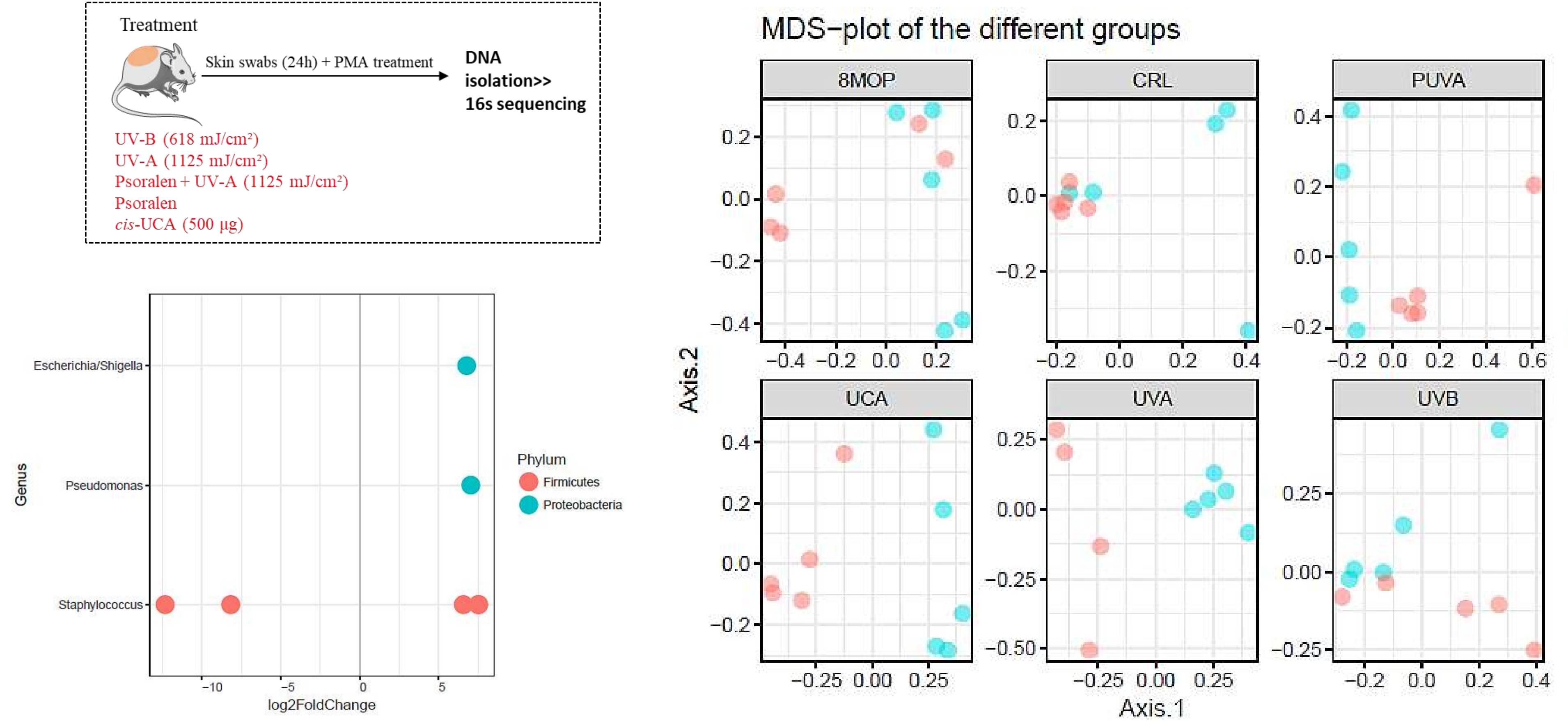

mePoint

After

Fig 3 (a): Modulation of skin microbiome by cis-UCA ( $500 \mu \mathrm{g}$ ) and UV-R at $24 \mathrm{~h}$. MDS plots show a distinct separation between skin microbiome before and after treatment, notably for cis-UCA. Furthermore cis-UCA significantly increases abundance of Pseudomonas, Escherichia/Shigella species.

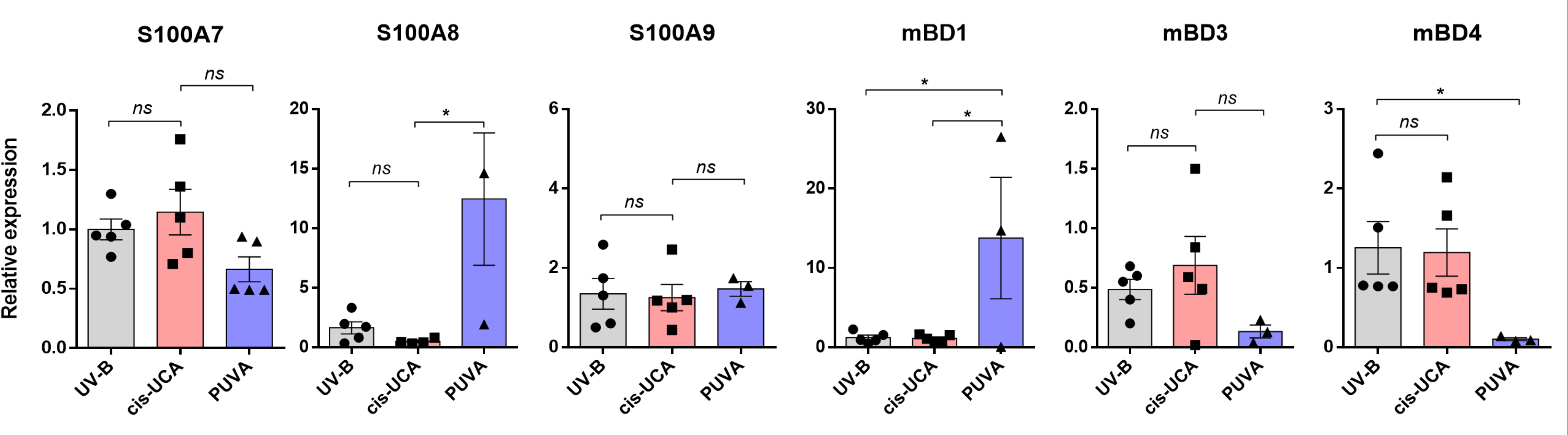

Fig 3 (b): AMPs gene signature significanlty differs at 24h. Expression of AMPs is reduced at $24 \mathrm{~h}$ after cis-UCA $(500 \mu \mathrm{g})$, whereas PUVA causes extreme variations in gene expression.

ANOVA test (One-Way) with Tukey post hoc test. * $p<0.05 ; * *$ p $<0.01 ; * * * p<0.001$

4. cis-UCA, PUVA and UV-B suppress immune reaction to contact allergen DNFB.

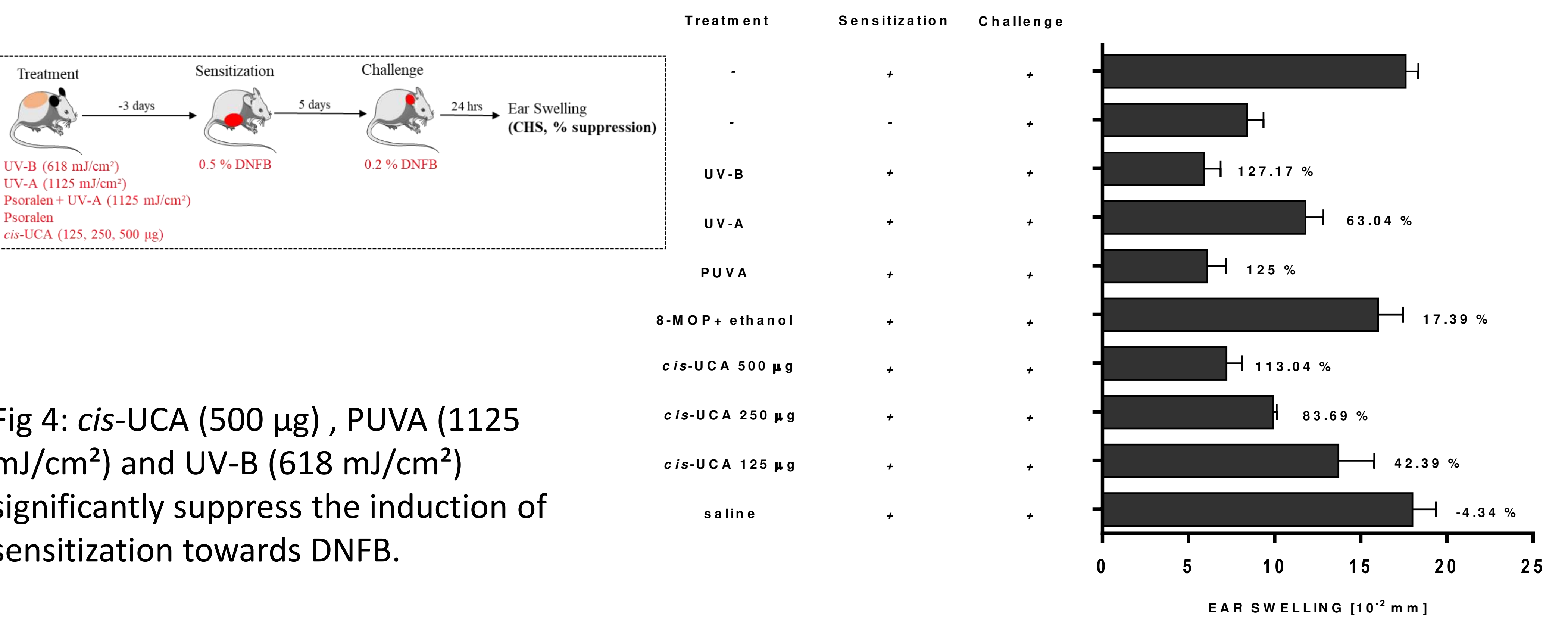

Summary and Conclusion:

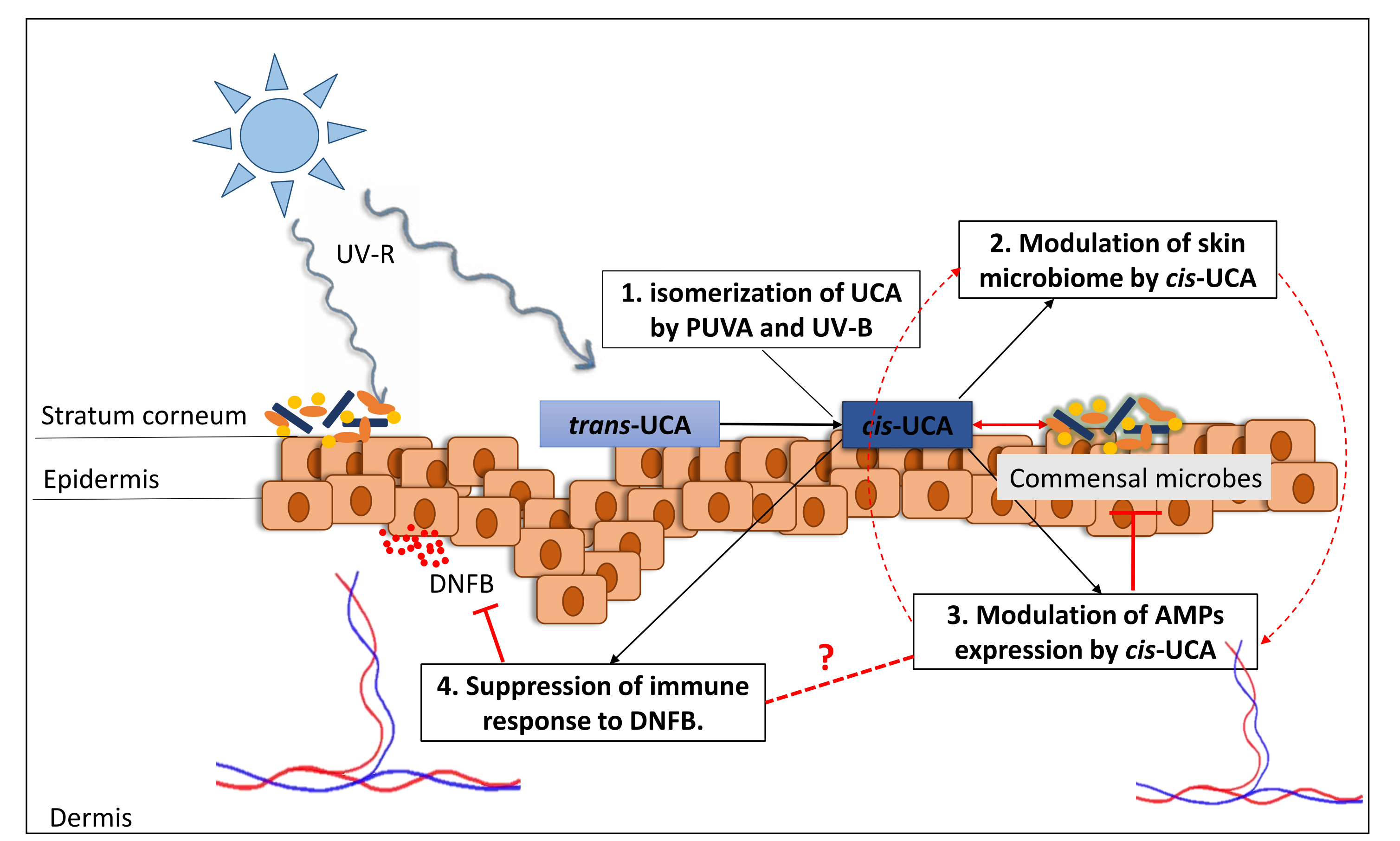

$\checkmark$ PUVA, UV-A and UV-B significantly increase the formation of cis-UCA.

$\checkmark$ Application of cis-UCA on the skin alters the microbial landscape and AMP gene expression of the skin.

$\checkmark$ There is dose-dependent increase in immune suppression against the contact allergen DNFB, when the mice are pre-treated with UV-B, PUVA or cis-UCA (but not UV-A).

$\checkmark$ Though UV-A significantly isomerizes trans-UCA, it shows no significant immune suppression to DNFB.

cis-UCA may have a role in immune suppression through affecting skin microbiome and AMPs expression. 